\title{
Parametric Instabilities of Interacting Bosons in Periodically Driven 1D Optical Lattices
}

\author{
K. Wintersperger, ${ }^{1,2, \dagger}$ M. Bukov, ${ }^{3, \dagger}$ J. Näger, ${ }^{1,2}$ S. Lellouch, ${ }^{4,5}$ E. Demler, ${ }^{6}$ U. Schneider, ${ }^{7}$ \\ I. Bloch, ${ }^{1,2,8}$ N. Goldman, ${ }^{4}$ and M. Aidelsburger $\oplus^{1,2, *}$ \\ ${ }^{1}$ Fakultät für Physik, Ludwig-Maximilians-Universität München, Schellingstraße 4, 80799 München, Germany \\ ${ }^{2}$ Munich Center for Quantum Science and Technology (MCQST), Schellingstraße 4, 80799 München, Germany \\ ${ }^{3}$ Department of Physics, University of California, Berkeley, California 94720, USA \\ ${ }^{4}$ Center for Nonlinear Phenomena and Complex Systems, Université Libre de Bruxelles, \\ CP 231, Campus Plaine, 1050 Brussels, Belgium \\ ${ }^{5}$ Laboratoire de Physique des Lasers, Atomes et Molécules, Université Lille 1 Sciences et Technologies, \\ CNRS, 59655 Villeneuve d'Ascq Cedex, France \\ ${ }^{6}$ Department of Physics, Harvard University, Cambridge, Massachusetts 02138, USA \\ ${ }^{7}$ Cavendish Laboratory, University of Cambridge, J.J. Thomson Avenue, Cambridge CB3 OHE, United Kingdom \\ ${ }^{8}$ Max-Planck-Institut für Quantenoptik, Hans-Kopfermann-Straße 1, 85748 Garching, Germany
}

(Received 24 August 2018; revised manuscript received 23 September 2019; accepted 12 December 2019; published 11 February 2020)

\begin{abstract}
Periodically driven quantum systems are currently explored in view of realizing novel many-body phases of matter. This approach is particularly promising in gases of ultracold atoms, where sophisticated shaking protocols can be realized and interparticle interactions are well controlled. The combination of interactions and time-periodic driving, however, often leads to uncontrollable heating and instabilities, potentially preventing practical applications of Floquet engineering in large many-body quantum systems. In this work, we experimentally identify the existence of parametric instabilities in weakly interacting Bose-Einstein condensates in strongly driven optical lattices through momentum-resolved measurements, in line with theoretical predictions. Parametric instabilities can trigger the destruction of weakly interacting Bose-Einstein condensates through the rapid growth of collective excitations, in particular in systems with weak harmonic confinement transverse to the lattice axis. Understanding the onset of parametric instabilities in driven quantum matter is crucial for determining optimal conditions for the engineering of modulation-induced many-body systems.
\end{abstract}

DOI: $10.1103 /$ PhysRevX.10.011030

Floquet engineering has proven to be a powerful technique for the design of novel quantum systems with tailored properties, unattainable in conventional static systems [1-3]. It is based on the design of time-periodic systems, whose stroboscopic evolution is governed by an effective time-independent Hamiltonian featuring the desired properties. Floquet engineering is captivating due to its conceptual simplicity and its potentially farreaching applications for engineering novel states of matter. For instance, it has been used to manipulate the electronic properties of solid-state systems [4-7], to realize time crystals $[8,9]$, to engineer artificial magnetic

\footnotetext{
*Corresponding author. monika.aidelsburger@physik.uni-muenchen.de

These authors contributed equally to this work.

Published by the American Physical Society under the terms of the Creative Commons Attribution 4.0 International license. Further distribution of this work must maintain attribution to the author(s) and the published article's title, journal citation, and DOI.
}

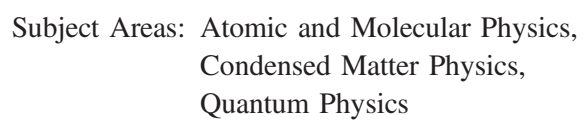

fields and topological Bloch bands in cold atoms [10-15], photonics [16-18], and superconducting circuits [19], to generate density-dependent gauge fields [20,21], and to explore the rich physics of lattice gauge theories [22].

The complex interplay between periodic driving and interactions poses theoretical and experimental challenges. In time-periodic systems, energy conservation is relaxed due to the possibility to absorb and emit energy quanta from the drive, and any driven ergodic system is expected to eventually heat up to infinite temperature $[23,24]$. Recent experiments [25-29] have addressed this problem for interacting atoms in shaken optical lattices. In particular, it has been shown [27,30-33] that heating rates are well captured by a Floquet Fermi's golden rule (FFGR) approach, if they are evaluated at sufficiently long times. This approach suggests that the long-time dynamics is dominated by incoherent two-body scattering processes [32]. In contrast, the onset of heating in bosonic systems is expected to be triggered by coherent processes, in the form of parametric instabilities [34-37].

First evidence for parametric instabilities has been found indirectly via spectroscopic measurements, in 
(a)

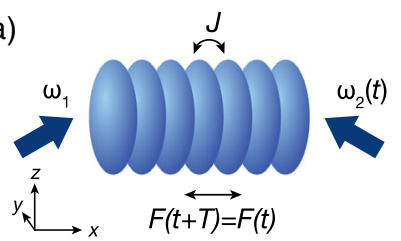

(b)

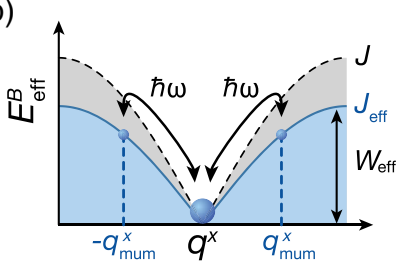

(c)

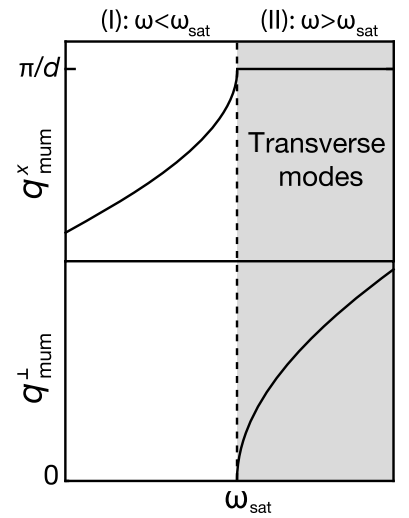

FIG. 1. Illustrations of the experimental setup and the properties of parametric instabilities. (a) Schematic of the driven 1D optical lattice with tunneling $J$, generated by two laser beams with frequencies $\omega_{1,2}$, and weak harmonic transverse confinement. Modulating $\omega_{2}(t)$ periodically with frequency $\omega$ generates a force $F(t)$ with period $T=2 \pi / \omega$. (b) Illustration of the effective 1D Bogoliubov dispersion $E_{\text {eff }}^{B}\left(q^{x}, J_{\text {eff }}\right)$, with width $W_{\text {eff }}$ reduced according to the Floquet-renormalized tunnel coupling $J_{\text {eff }}$ (blue) compared to the static lattice case (gray). Parametric resonances at $E_{\mathrm{eff}}^{B}=\hbar \omega$ induced by the modulation lead to instabilities centered around the most unstable mode $q_{\text {mum }}^{x} ; \hbar=h /(2 \pi)$ is the reduced Planck's constant. (c) Momentum of the most unstable mode, $\mathbf{q}_{\text {mum }}=\left(q_{\text {mum }}^{x}, \mathbf{q}_{\text {mum }}^{\perp}\right)$, as predicted by Bogoliubov theory, which shows a clear separation between lattice (I) and transverse (II) degrees of freedom (d.o.f.), that occurs at the saturation frequency $\hbar \omega_{\text {sat }} \approx W_{\text {eff }} ; d$ is the lattice constant.

amplitude-modulated optical lattices [34,38]. In this work, we directly reveal the existence and nature of parametric instabilities by measuring the momentum distribution of weakly interacting bosons in a periodically driven onedimensional (1D) optical lattice [Fig. 1(a)]. These instabilities exist whenever the energy quantum $\hbar \omega$ associated with the drive matches the energy of a collective excitation, as dictated by the effective Bogoliubov dispersion $E_{\text {eff }}^{B}\left(\mathbf{q}, J_{\text {eff }}\right)$, where $\mathbf{q}$ denotes the momentum of the excitation and $J_{\text {eff }}$ is the effective tunnel coupling renormalized by the drive [39] [Fig. 1(b)]. In a strictly 1D lattice, the strongest instability occurs at the two-photon resonance [37], $2 \hbar \omega=2 E_{\text {eff }}^{B}\left(q_{\text {res }}^{x}, J_{\text {eff }}\right)$. In the single-band approximation, there exists a stable parameter regime without parametric instabilities for $\omega>\omega_{\text {sat }} \approx W_{\text {eff }} / \hbar$, where $W_{\text {eff }}=\sqrt{4\left|J_{\text {eff }}\right|\left(4\left|J_{\text {eff }}\right|+2 g\right)}$ is the bandwidth of the effective Bogoliubov dispersion and $g$ is the interaction energy (see Ref. [37] and the Appendix A). In a 3D system with weak harmonic confinement transverse to the lattice axis [Fig. 1(a)], however, no stable parameter regime exists [27,31-34,37] and parametric instabilities occur via the closely spaced transverse modes [34,37,38] [regime (II) in Fig. 1(c)]. Moreover, our numerical simulations indicate that a harmonic confinement along

the lattice axis further prevents the existence of a stable region, even in a true $1 \mathrm{D}$ geometry (Appendix B).

The appearance of instabilities is common in static weakly interacting bosonic lattice systems. For instance, Landau instabilities can occur when the condensate is prepared at a finite quasimomentum, where the effective mass in the band structure is negative; such configurations can also display dynamical instabilities, where Bogoliubov excitations grow exponentially [40-46]. We emphasize that the origin of such instabilities is different compared to those revealed in this work. These instabilities exist even for a condensate initially at rest; in contrast, parametric instabilities originate from the time-dependent nature of the drive. We note that instabilities are not necessarily detrimental but can result in interesting phenomena, such as parametric amplification, four-wave mixing [44-47], and pattern formation [48-51].

The experiment starts by loading an almost pure BoseEinstein condensate (BEC) of about $N=3.7(4) \times 10^{5}{ }^{39} \mathrm{~K}$ atoms within $100 \mathrm{~ms}$ into a $1 \mathrm{D}$ optical lattice aligned along the $x$ axis, with lattice constant $d=425 \mathrm{~nm}$ and depth $V_{\text {lat }}=11.0(3) E_{R}$, where $E_{R}=h^{2} /\left(8 m d^{2}\right)=h \times 7.1 \mathrm{kHz}$ is the recoil energy and $m$ the mass of an atom. Additional confinement is provided by an optical dipole trap. The harmonic trapping frequencies of the combined potential are $\omega_{r} /(2 \pi)=26(2) \mathrm{Hz}$ in the $x y$ plane and $\omega_{z} /(2 \pi)=$ 204(3) Hz in the vertical direction. The lattice is created by interfering two laser beams with $\lambda=736.8 \mathrm{~nm}$ under an angle of $120^{\circ}$. Its position is modulated by varying the frequency of one lattice laser beam [Fig. 1(a)], $\omega_{2}(t)=$ $\omega_{1}+2 \pi f \sin (\omega t+\varphi)$, where $\varphi$ is the phase of the drive and $f$ is the amplitude of the phase modulation. The lattice modulation is turned on suddenly in order to be able to observe the presence of collective excitations after few modulation periods (we verified that ramping up the modulation amplitude within five cycles does not modify our main results). We hold the atoms in the modulated lattice for integer multiples of the driving period and determine the momentum distribution by performing band mapping (the modulation is turned off abruptly followed by a $100-\mu$ s-long linear ramp-down of the lattice) and subsequent time-of-flight (TOF) imaging [Fig. 2(a)].

In the reference frame of the lattice, the modulation leads to a time-varying force, $F(t)=F_{0} \cos (\omega t+\varphi)$, with $F_{0}=m d f \omega$ [39], and the time-dependent tight-binding Hamiltonian describing the dynamics takes the form

$$
\begin{aligned}
\hat{H}(t)= & \int_{\mathbf{r}_{\perp}}\left[-J \sum_{\langle i j\rangle}\left(\hat{a}_{i, \mathbf{r}^{\perp}}^{\dagger} \hat{a}_{j, \mathbf{r}^{\perp}}+\hat{a}_{j, \mathbf{r}^{\perp}}^{\dagger} \hat{a}_{i, \mathbf{r}^{\perp}}\right)\right. \\
& +\frac{U}{2} \sum_{j} \hat{n}_{j, \mathbf{r}^{\perp}}\left(\hat{n}_{j, \mathbf{r}^{\perp}}-1\right) \\
& \left.+K \cos (\omega t+\varphi) \sum_{j} j \hat{n}_{j, \mathbf{r}^{\perp}}+\hat{H}^{\perp}+\hat{H}_{\mathrm{harm}}\right],
\end{aligned}
$$



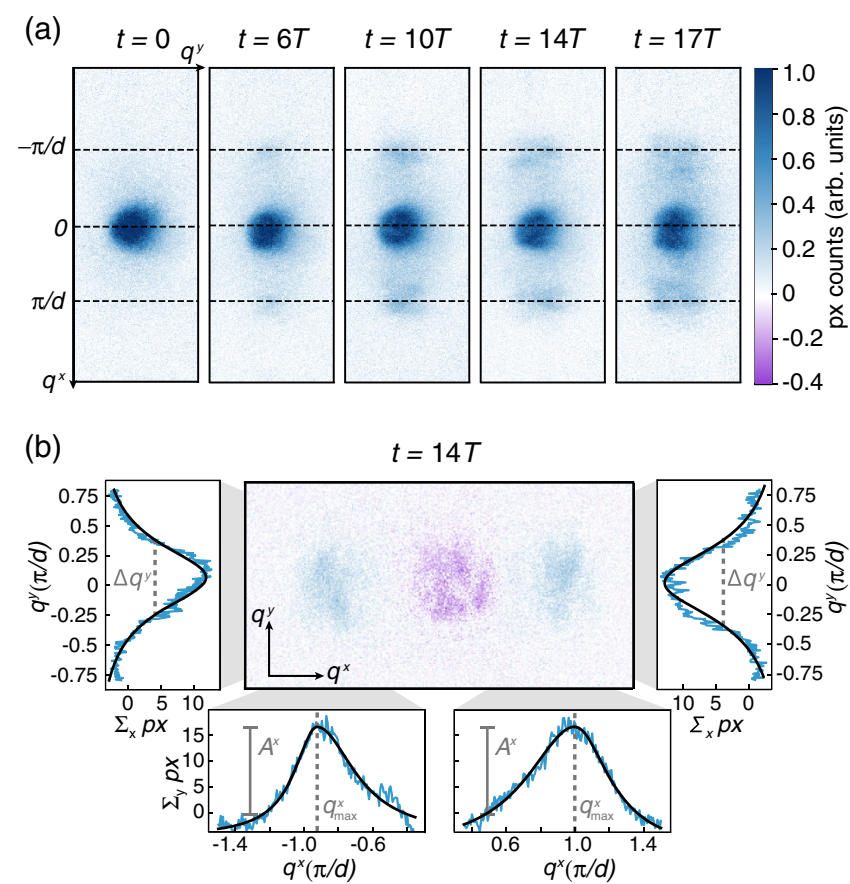

FIG. 2. Momentum-resolved images of the most unstable mode for $\alpha=1.78, \omega /(2 \pi)=720 \mathrm{~Hz}$, and $\varphi=0$. (a) Time series of absorption images after $6 \mathrm{~ms}$ TOF. The edges and the center of the first Brillouin zone (BZ) are marked by dashed lines. (b) Difference image at $t=14 T$, obtained by subtracting the condensate profile at $t=0$. The lower panels show the 1D profiles along $q^{x}$, resulting from summation of the difference image perpendicular to the lattice axis for the left and right excitation peak together with asymmetric Lorentzian fits (black solid lines), used to determine the amplitude $A^{x}$ and position $q_{\max }^{x}$ (dashed lines) of the excitation peaks [52]. The left- and righthand plots show the profiles resulting from summation along the lattice axis with Lorentzian fits to determine the transverse full width at half maximum $\Delta q^{y}$ (dashed lines) of the peaks. Here, we integrate the density profile in a region of interest containing the left or right peak only.

where $\hat{a}_{i, \mathbf{r}^{\perp}}^{\dagger}$ and $\hat{a}_{i, \mathbf{r}^{\perp}}$ are the bosonic creation and annihilation operators on lattice site $i$ and transverse position $\mathbf{r}^{\perp}, \hat{n}_{i, \mathbf{r}^{\perp}}$ is the corresponding number operator, $J / h=$ $108(7) \mathrm{Hz}$ is the tunnel coupling, $U$ is the on-site interaction, $K=F_{0} d, \hat{H}^{\perp}$ denotes the kinetic energy along the transverse direction, and $\hat{H}_{\text {harm }}$ is the $3 \mathrm{D}$ harmonic confinement. The Feshbach resonance of ${ }^{39} \mathrm{~K}$ at 403.4(7)G enables us to work in the weakly interacting regime at the scattering length $a_{s}=20 a_{0}$ (with $a_{0}$ the Bohr radius), where a mean-field approach is expected to be valid. The Feshbach resonance is crucial in order to find experimental parameter regimes, where parametric instabilities could be clearly identified in momentumspace images. Additional nonlinear effects rapidly counteract the exponential growth of the instabilities (see Ref. [37] and Appendix C), leaving only a small window of suitable parameters to observe it.
In the noninteracting limit, Floquet theory predicts that the dynamics is well described by a time-independent Hamiltonian, with renormalized tunnel coupling, $J_{\text {eff }}=$ $J \mathcal{J}_{0}(\alpha)$ [1-3,39]. Here $\mathcal{J}_{\nu}$ is the $\nu$ th-order Bessel function of the first kind and $\alpha=K /(\hbar \omega)$. Note that $\alpha$ is independent of the modulation frequency $\omega$. The measurements are performed in a strong-driving regime, $1<$ $\alpha<2$, where the effects of the drive are nonperturbative, while the minimum of the effective dispersion remains at $q^{x}=0[39,46]$. To avoid single-particle interband resonances [61], the modulation frequency is chosen well below the first single-particle band gap of the static lattice, $\Delta_{21} / h=41.6(5) \mathrm{kHz}$. Indeed, we do not observe excitations to higher bands during the measurements, which would be visible in the TOF images [Fig. 2(a)].

In order to identify instabilities in the system, we monitor the appearance of collective excitations by measuring the momentum distribution of the atoms at stroboscopic times, $t=\ell T, \ell \in \mathbb{N}$, for various modulation frequencies and amplitudes. We find that after few modulation periods a small fraction of atoms is excited into additional momentum components that are distinct from the initial condensate at $q^{x}=0$ [Fig. 2(a)]. The amplitude of these modes grows as a function of the modulation time and excitation peaks eventually start to broaden after long modulation times [see $t=17 T$ in Fig. 2(a)] due to saturation and nonlinear effects (Appendix C). We note that the micromotion leads to additional oscillations in the occupation of the excitations at nonstroboscopic times.

We attribute the observed excitations to parametric instabilities that occur whenever a resonance exists between the energy associated with one drive quantum $\hbar \omega$ and the Bogoliubov spectrum $E_{\text {eff }}^{B}(\mathbf{q})$ [see Eq. (A2) in Appendix A] of collective excitations [34,35,37]. In the presence of transverse modes there is no stable parameter regime, since the transverse kinetic energy has no upper bound. There is always a set of resonant excitations determined by the resonance condition

$$
\hbar \omega=E_{\mathrm{eff}}^{B}\left(\mathbf{q}_{\mathrm{res}}\right), \quad \mathbf{q}_{\mathrm{res}}=\left(q_{\mathrm{res}}^{x}, \mathbf{q}_{\mathrm{res}}^{\perp}\right),
$$

which causes the system to be necessarily unstable. Each collective mode grows at a different rate $\Gamma_{\mathbf{q}}$, and we denote the one associated with the dominating growth rate, $\Gamma=$ $\max _{\mathbf{q}} \Gamma_{\mathbf{q}}$, the most unstable mode ("mum") with the corresponding momentum $\mathbf{q}_{\text {mum }}$.

For simplicity, we henceforth neglect the 3D harmonic confinement $\hat{H}_{\text {harm }}$ in our theoretical analysis and set the transverse kinetic term equal to a free-particle kinetic energy, $\hat{H}^{\perp}=\sum_{j, \mathbf{q}^{\perp}}\left[\left(\hbar \mathbf{q}^{\perp}\right)^{2} / 2 m\right] \hat{n}_{j, \mathbf{q}^{\perp}}$. For weak harmonic confinement, where the transverse modes are closely spaced, we expect this to be a good approximation. We note, however, that the harmonic confinement along the lattice axis largely modifies the stability of a strictly 1D system, where it prevents the existence of a true stable 
parameter regime, as the energy spectrum becomes unbounded (Appendix B).

Following the approach developed in Refs. [35,37] based on the time-dependent Bogoliubov-de Gennes (BdG) equations of motion [52], we find approximate analytic solutions for the momentum $\mathbf{q}_{\text {mum }}$ and the growth rate $\Gamma$ of the most unstable mode (see Ref. [37] and Appendix A). To lowest order in this perturbative treatment, there is a clear separation between the lattice and transverse d.o.f., resulting in two distinct regimes [Fig. 1(c)]:

(I) $\omega<\omega_{\text {sat }}: q_{\text {mum }}^{x}<\pi / d$ and $\left|\mathbf{q}_{\text {mum }}^{\perp}\right|=0$,

(II) $\omega>\omega_{\text {sat }}: q_{\text {mum }}^{x}=\pi / d$ and $\left|\mathbf{q}_{\text {mum }}^{\perp}\right|>0$.

In the first regime (I), the modulation mainly couples to excitations along the lattice direction,

$$
q_{\mathrm{mum}}^{x}=2 \arcsin \sqrt{\left[\sqrt{g^{2}+(\hbar \omega)^{2}}-g\right] /\left(4 J_{\mathrm{eff}}\right)} / d,
$$

and the instability is not affected by the transverse modes $\left(\left|\mathbf{q}_{\text {mum }}^{\perp}\right|=0\right)$; here $g=n U$ denotes the interaction energy and $n$ is the mean density (see Appendix B for a discussion of the inhomogeneous density profile of a harmonically trapped gas). When reaching the second regime (II), in contrast, the transverse d.o.f. dominate; $\mathbf{q}_{\text {mum }}^{\perp}$ becomes finite and grows according to

$$
\hbar^{2}\left(\mathbf{q}_{\text {mum }}^{\perp}\right)^{2} /(2 m)=\sqrt{g^{2}+(\hbar \omega)^{2}}-g-4 J_{\text {eff }} .
$$

To quantify the position of the most unstable mode experimentally from the TOF images [Fig. 2(a)], we subtract the mean initial condensate profile at $t=0$ from each individual TOF image, measured at $t>0$. A typical result is shown in Fig. 2(b) (a more detailed description of the data analysis can be found in the Supplemental Material [52]). To characterize the excitations along the lattice direction $q_{\mathrm{mum}}^{x}$, we integrate the $2 \mathrm{D}$ difference profile perpendicular to the lattice axis [lower panels in Fig. 2(b)]. The resulting 1D profile is divided into two parts excluding the negative part at small $\left|q^{x}\right|$ (purple), which arises due to the small depletion of the condensate. We determine the position $q_{\max }^{x}$ and amplitude $A^{x}$ of the excitation peaks by fitting asymmetric Lorentzian functions to each peak [Eq. (S.1) in the Supplemental Material [52] ].

The transverse momentum component $\mathbf{q}_{\text {mum }}^{\perp}$ of the most unstable mode is masked by the initial momentum spread of the condensate and the width of the parametric resonance. Nevertheless, it manifests itself in a broadening, which we monitor by extracting the transverse width $\Delta q^{y}$ of the excitation peaks: We integrate the 2D difference profile along the lattice direction using a region of interest that contains only the left or the right peak. We fit the resulting 1D profiles [left- and right-hand panel in Fig. 2(b)] with a symmetric Lorentzian and extract the full width at half maximum. The fit parameters obtained for the left and right (a)

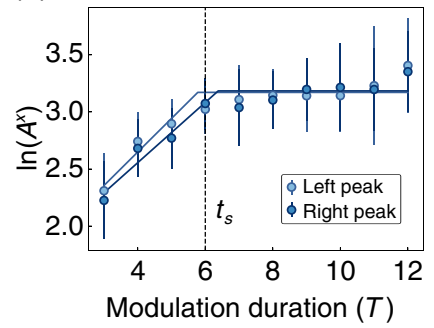

(b)

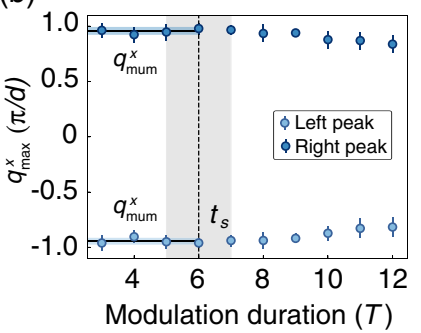

FIG. 3. Time-resolved growth of the most unstable mode for $\alpha=1.78$ and $\omega /(2 \pi)=720 \mathrm{~Hz}$. (a) Logarithmic plot of the peak amplitude $A^{x}$ as a function of modulation time. The solid lines are fits to the data to extract the end of the short-time regime $t_{s}$ (black dashed line). (b) Position of the peak maximum $q_{\max }^{x}$ along $q^{x}$ as a function of modulation time. The most unstable mode $q_{\text {mum }}^{x}$ is defined as the weighted mean of all $q_{\max }^{x}$ for $t \leq t_{s}$. Its value is shown as the solid black line; the shaded blue bars denote its standard error of the weighted mean. The shaded gray area defines the range of hold times over which the transverse width $\Delta q^{y}$ is averaged. Each data point is an average over $\sim 10$ individual experimental realizations; error bars indicate the standard deviation. To minimize systematic deviations in the band-mapping images, we average $\lesssim 5$ realizations for a modulation phase $\varphi=0$ and $\lesssim 5$ with $\varphi=\pi$ (Fig. S1 of Supplemental Material [52]).

peak are then averaged over all images for each modulation time $t>0$.

In Fig. 3 we show a typical time series for the position $q_{\max }^{x}$ and amplitude $A^{x}$ of the dominant mode (full dataset is shown in the Supplemental Material [52]). While there are remnants of a moderate exponential growth at short times $t<t_{s}$, we predominantly find an explicitly timedependent growth rate. This is due to incoherent processes caused by interactions between collective excitations and between the excited modes and the condensate [37]. This behavior is confirmed by our numerical simulations beyond BdG theory discussed in Appendix C. In order to quantitatively compare our experimental data with analytical expressions obtained using Bogoliubov theory, we restrict the analysis to a small time window $t<t_{s}$ before saturation and nonlinear effects start to dominate. This is motivated by the good agreement between numerical simulations and analytical formulas for short times (Fig. 9 in Appendix C). The parameter $t_{s}$ is evaluated by fitting a piecewise function consisting of a linear and a constant part to $\ln \left(A^{x}\right)$ individually for the left and right excitation peak [Fig. 3(a)] and averaging the two results [52]. The corresponding growth rates (for $t<t_{s}$ ) are in good agreement with Bogoliubov theory (Fig. 10 in Appendix C), validating this approach. The position of the most unstable mode $q_{\text {mum }}^{x}$ is then defined as the average over the peak positions $q_{\max }^{x}$ for all modulation times $t<t_{s}$. We observe a decrease of $q_{\max }^{x}$ at modulation times $t>t_{s}$ indicating the onset of additional scattering events not captured by Bogoliubov theory. In order to 


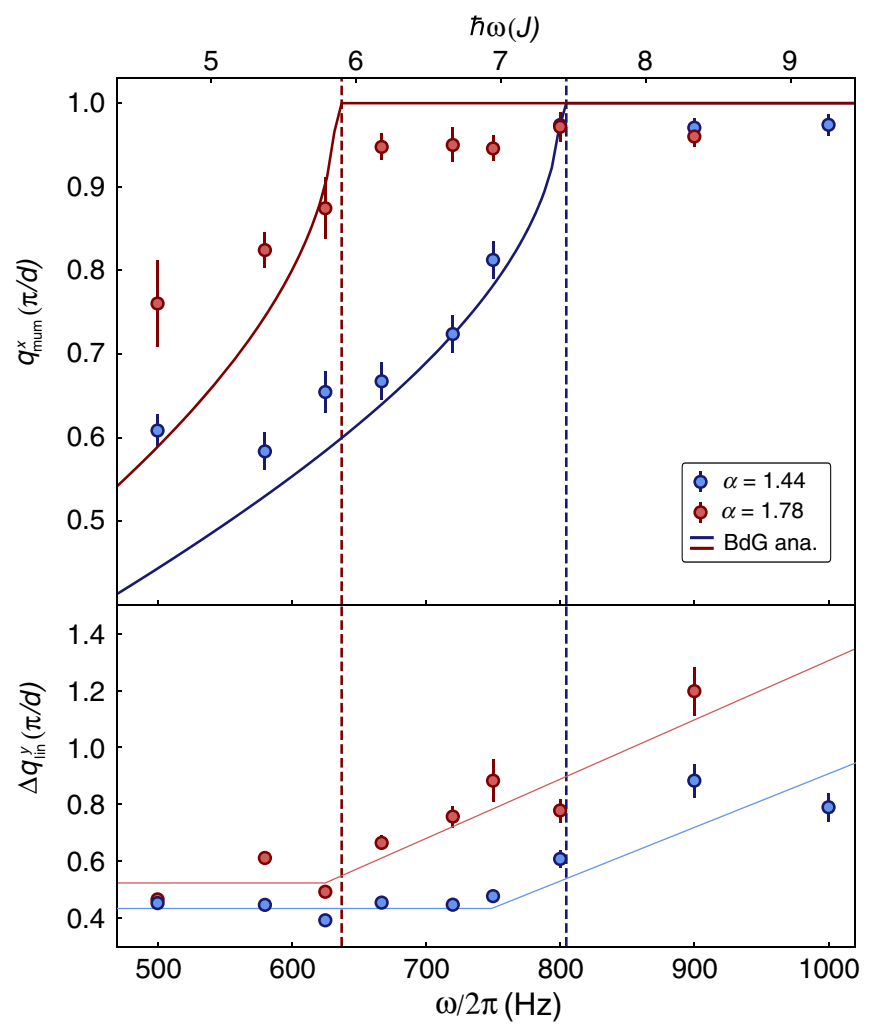

FIG. 4. Position of the most unstable mode and influence of transverse modes. Upper panel: Position of the most unstable mode $q_{\text {mum }}^{x}$ as a function of modulation frequency for $\alpha=1.44$ and $\alpha=1.78$. The error bars denote the standard error of the mean. The solid lines show Eq. (3) for $g=11.5 \mathrm{~J}$. The vertical dashed lines mark the corresponding saturation frequency. Lower panel: Transverse width $\Delta q_{\text {lin }}^{y}(\omega)$. Error bars denote the standard error of the weighted mean. The thin lines are guides to the eye.

study the behavior along the transverse direction, we analyze the peak width $\Delta q^{y}$ at the end of the short-time regime, where the amount of transverse excitations is expected to be maximal (full dataset is shown in the Supplemental Material [52]). In order to reduce statistical errors, we average $\Delta q^{y}$ over modulation times between $t_{s}-1$ and $t_{s}+1$ in units of the modulation period [Fig. 3(b)] to obtain the experimental value for the width $\Delta q_{\text {lin }}^{y}[52]$.

The position of the most unstable mode along the lattice axis $q_{\text {mum }}^{x}$ and the transverse width $\Delta q_{\text {lin }}^{y}$ is measured for various modulation parameters around the saturation frequency, where we expect a crossover between parametric instabilities dominated by excitations along the lattice axis [regime (I)] and those that are facilitated by the presence of transverse modes [regime (II)]. The results are shown in Fig. 4.

We find that $q_{\mathrm{mum}}^{x}$ indeed increases with $\omega$ until it saturates at $q_{\text {mum }}^{x} \approx \pi / d$, the edge of the Brillouin zone, at a frequency $\omega_{\text {sat }}$ that depends on the driving amplitude $\alpha$ (Fig. 4, upper panel). The small deviation of the measured positions from $\pi / d$ is mainly due to the short TOF used in the experiment [52]. The saturation frequency $\omega_{\text {sat }}$ calculated from Eq. (3) matches the experimental data well for an interaction parameter $g=11.5 \mathrm{~J}$. From our measured in situ density profiles we obtain an interaction parameter $g_{\max } \approx$ $8 J$ in the center of the trap. The deviation is most likely due to a systematic uncertainty in the atom number calibration [52]. At the same time, we observe that the width $\Delta q_{\text {lin }}^{y}(\omega)$ transverse to the lattice axis starts to increase with frequency for $\omega>\omega_{\text {sat }}(\alpha)$, simultaneously with the saturation of $q_{\text {mum }}^{x}$ at the BZ edge (Fig. 4, lower panel), as expected from lowest-order perturbation theory Eqs. (3) and (4). Moreover, the transition point $\omega_{\text {sat }}(\alpha)$ decreases for larger driving parameters $\alpha$, in line with the reduction of $J_{\text {eff }}$.

We further observe that the shape of the $q_{\text {mum }}^{x}$ curve differs from the theoretical prediction for low modulation frequencies, in particular for the large modulation amplitude $\alpha=1.78$. We attribute this deviation mainly to the following effects. First, the short-time window, during which we can clearly identify the most unstable mode, strongly depends on the modulation parameters. The time $t_{s}$, which marks the end of the short-time window, decreases with decreasing modulation frequency and increasing modulation amplitude (Fig. S2 of Ref. [52]). This complicates the identification of the peak maxima. If $t_{s}$ becomes too small, there is not enough time for the most unstable mode to dominate over the other excited modes. Second, the initial width of the momentum distribution of the condensate at $t=0$ poses a fundamental limitation that prevents us from measuring positions smaller than $\approx 0.4 \pi / d$. Moreover, for large modulation amplitudes, higher-order corrections to the analytical formulas Eqs. (3) and (4) become relevant (Appendix A).

In summary, we have demonstrated the first direct evidence for parametric instabilities in shaken optical lattices via momentum-resolved measurements. Our experiments were performed with a large 3D system of weakly interacting bosons, where exact numerical calculations including the harmonic trap are not feasible. By tuning the scattering length with a Feshbach resonance, we were able to identify and address experimental parameter regimes where the short-time dynamics is well described by BdG theory. While the instability rates are time dependent due to competing processes that dominate on different timescales, the momentum of the most unstable mode turned out to be a reliable observable. The obtained results are in agreement with the analytic approach derived in Ref. [37], which enables the development of an intuitive understanding and allows us to identify stable parameter regimes in driven lattice models from simple energetic arguments. We were able to verify the existence of key bottlenecks in current experimental settings with weak transverse confinement $[10,11,14,26,62-64]$ that need to be overcome by freezing the transverse d.o.f. and generating a box-type longitudinal confinement $[65,66]$. Parametric instabilities can indeed lead to a depletion of the condensate, where subsequent scattering 
events result in large heating rates. Our results are of strong interest for future experiments based on Floquet engineering [3], as they indicate the necessity to engineer full 3D lattice systems, where stable regimes can be found [35,37]. Parametric resonances are expected to be present whenever the $\mathrm{BdG}$ equations of motion include time-periodic features, and hence may turn out to play an important role in a wide family of Floquet-engineered systems, such as periodically driven superfluids [3] and superconductors [67], photonic devices $[68,69]$, and also in the context of cosmology [70].

\section{ACKNOWLEDGMENTS}

We thank T. Boulier, C. Braun, M. Cheneau, C. Chin, T. Esslinger, L. Fallani, D. Guéry-Odelin, A. Polkovnikov, T. Porto, M. Reitter, L. Tarruell, and D. Sels for insightful discussions. The work in Munich was supported by the Deutsche Forschungsgemeinschaft (FOR2414 Grant No. BL 574/17-1), the European Commission (UQUAM Grant No. 5319278, AQuS), the Nanosystems Initiative Munich (NIM) Grant No. EXC4, and by the Deutsche Forschungsgemeinschaft (DFG, German Research Foundation) under Germany's Excellence Strategy-EXC2111-39081486. The work in Brussels was financed by the FRS-FNRS (Belgium) and the TopoCold ERC Starting Grant. U.S. acknowledges support from the EPSRC Programme Grant DesOEQ (EP/P009565/1). M. B. acknowledges support from the Emergent Phenomena in Quantum Systems initiative of the Gordon and Betty Moore Foundation, and the U.S. Department of Energy, Office of Science, Office of Advanced Scientific Computing Research, Quantum Algorithm Teams Program. E. D. was supported by Harvard-MIT CUA, NSF Grant
No. DMR-1308435, AFOSR Quantum Simulation MURI, AFOSR-MURI Photonic Quantum Matter (Grant No. FA95501610323). We used Quspin [71,72] to perform the numerical simulations. The authors are pleased to acknowledge that the computational work reported on in this paper was performed on the Shared Computing Cluster which is administered by Boston University's Research Computing Services.

Note added.-Recently, short-time heating rates, which are expected to be dominated by parametric instabilities, have been investigated in modulated 2D lattices [73].

\section{APPENDIX A: ANALYTICAL TREATMENT OF PARAMETRIC INSTABILITIES WITHIN THE BOGOLIUBOV APPROXIMATION}

Here, we recall the analytical method developed in Ref. [35] to extract the instability properties of the system within the Bogoliubov approximation. As mentioned in the main text, for simplicity, we neglect the harmonic confinement, so that the transverse kinetic energy is determined by the free-particle dispersion relation. It has been shown that the Bogoliubov equations of motion [Eq. (S.9) in Sec. S3 A of the Supplemental Material [52]] can be mapped to a parametric oscillator model [36,74], a seminal model of the periodically driven harmonic oscillator known to display parametric instabilities as soon as the drive frequency approaches twice the natural frequency. To see that, one should perform a series of suitable changes of basis and reference frames [37]. Applying the rotating wave approximation (RWA), we keep the leading-order harmonic and recast the Bogoliubov equations into the form

$$
i \hbar \partial_{t}\left(\begin{array}{c}
\tilde{u}_{\mathbf{q}}^{\prime} \\
\tilde{v}_{\mathbf{q}}^{\prime}
\end{array}\right)=\left[E_{\mathrm{eff}}^{B}(\mathbf{q}) \hat{\mathbf{1}}+\frac{A_{\mathbf{q}} E_{\mathrm{eff}}^{B}(\mathbf{q})}{2}\left(\begin{array}{cc}
0 & \cos (2 \omega t) e^{-2 i E_{\mathrm{eff}}^{B}(\mathbf{q}) t / \hbar} \\
-\cos (2 \omega t) e^{2 i E_{\mathrm{eff}}^{B}(\mathbf{q}) t / \hbar} & 0
\end{array}\right)\right]\left(\begin{array}{c}
\tilde{u}_{\mathbf{q}}^{\prime} \\
\tilde{v}_{\mathbf{q}}^{\prime}
\end{array}\right),
$$

where $\hat{\mathbf{1}}$ is the identity matrix,

$$
E_{\text {eff }}^{B}(\mathbf{q})=\sqrt{\left[4\left|J_{\text {eff }}\right| \sin ^{2}\left(q^{x} d / 2\right)+\left(\hbar \mathbf{q}^{\perp}\right)^{2} / 2 m\right]\left[4\left|J_{\text {eff }}\right| \sin ^{2}\left(q^{x} d / 2\right)+\left(\hbar \mathbf{q}^{\perp}\right)^{2} / 2 m+2 g\right]}
$$

denotes the effective (time-averaged) Bogoliubov dispersion, and we introduced the amplitude

$$
A_{\mathbf{q}}=16 J \mathcal{J}_{2}(\alpha) \sin ^{2}\left(q^{x} d / 2\right) \frac{g}{\left[E_{\text {eff }}^{B}(\mathbf{q})\right]^{2}}
$$

For the sake of simplicity, we have here restricted ourselves to the dominant harmonic of the drive, and dropped all terms in
Eq. (A1) that are irrelevant regarding the occurrence of instabilities - a simplification that was rigorously established in Ref. [37].

Perturbation theory.-As can be seen from a RWA treatment of Eq. (A1), each momentum mode $\mathbf{q}$ will display a dynamical instability (characterized by an exponential growth of its population), whenever the drive frequency $\omega$ approximately matches its effective Bogoliubov energy 
$E_{\text {eff }}^{B}(\mathbf{q})$, i.e., $\hbar \omega \approx E_{\text {eff }}^{B}(\mathbf{q})$. The analytical method to extract the associated instability rate is detailed in Refs. [37,74] and relies on a perturbation theory formulated in both $A_{\mathbf{q}}$ (the small parameter of the expansion, which is assumed to be smaller than 1 for the expansion to converge) and the detuning from the resonance $\delta_{\mathbf{q}} \equiv \hbar \omega-E_{\text {eff }}^{B}(\mathbf{q})$. In brief, the findings are the following.

(1) Zeroth order. The instability occurs only on resonance, i.e., $\hbar \omega=E_{\text {eff }}^{B}(\mathbf{q})$ and the instability rate is given by

$$
\begin{aligned}
\Gamma_{\mathbf{q}}^{*} & =\frac{A_{\mathbf{q}} E_{\text {eff }}^{B}(\mathbf{q})}{4 h} \\
& =\frac{4 J}{h} \mathcal{J}_{2}(\alpha) \sin ^{2}\left(q^{x} d / 2\right) \frac{g}{\hbar \omega},
\end{aligned}
$$

for all $\mathbf{q}$ fulfilling the resonance condition $\hbar \omega=$ $E_{\text {eff }}^{B}(\mathbf{q})$ and is zero for all other modes. Interestingly, not all resonant modes necessarily have the same instability rate. We focus on the most unstable mode, which has the largest rate $\Gamma=\max _{\mathbf{q}} \Gamma_{\mathbf{q}}^{*}$, which results in the following rates for the two regimes introduced in the main text:

$$
\text { (I) } \begin{aligned}
\omega & <\omega_{\text {sat }}: \\
\Gamma & =\frac{1}{h}\left(\sqrt{g^{2}+(\hbar \omega)^{2}}-g\right)\left|\frac{\mathcal{J}_{2}(\alpha)}{\mathcal{J}_{0}(\alpha)}\right| \frac{g}{\hbar \omega},
\end{aligned}
$$

(II) $\omega>\omega_{\text {sat }}$ :

$$
\Gamma=\frac{4 J}{h}\left|\mathcal{J}_{2}(\alpha)\right| \frac{g}{\hbar \omega} .
$$

The corresponding momentum $\mathbf{q}_{\text {mum }}$ of the most unstable mode to lowest order in this pertubative treatment is defined in Eqs. (3) and (4) in the main text.

(2) First order. We find that instability does not only occur on resonance but still arises in a finite window around the resonance point. The associated instability rate is given by

$$
\Gamma_{\mathbf{q}}=\frac{A_{\mathbf{q}} E_{\mathrm{eff}}^{B}(\mathbf{q})}{4 h} \sqrt{1-\left(\frac{\delta_{\mathbf{q}}}{A_{\mathbf{q}} E_{\mathrm{eff}}^{B}(\mathbf{q})}\right)^{2}},
$$

if the argument of the square root is positive, and $\Gamma_{\mathbf{q}} \equiv$ 0 otherwise. It is maximal at resonance and decreases with the distance from resonance, until it vanishes at the edges of the instability domain $[37,74]$.

(3) Second order. We no longer have explicit analytical expressions, but we find that $\Gamma_{\mathbf{q}}$ is the solution of an implicit equation which can be numerically solved $[37,74]$. While the width of the resonance is unaffected, we obtain that the instability rate is no longer maximal on resonance, but that the maximum
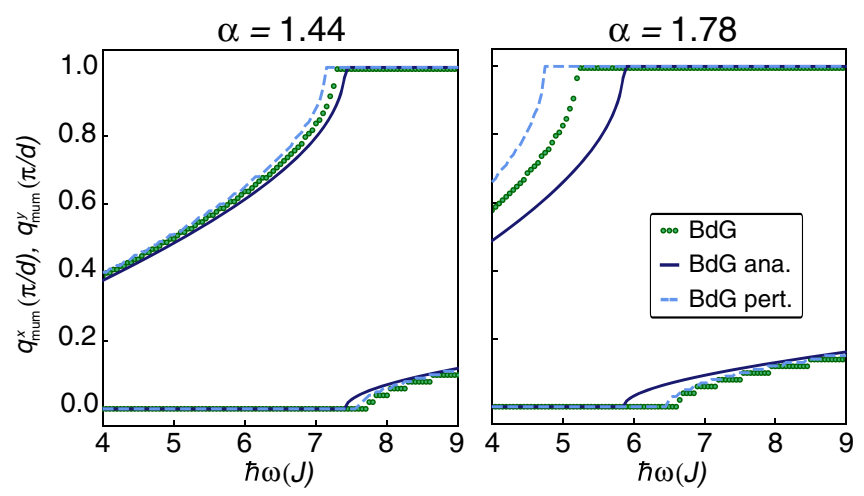

FIG. 5. Comparison of the perturbative analytic treatment calculated to zeroth [solid blue line, Eqs. (3) and (4)] and second order (dashed blue line) with numerical simulations of the exact BdG equations (green dots) for $g / J=11.5$ and two modulation amplitudes, $\alpha=1.44$ (left) and $\alpha=1.78$ (right). The steps in the numerical results are due to the discretization in momentum space.

instability point is slightly shifted from the resonance.

In Fig. 5 we show a comparison between the analytic formulas Eqs. (3) and (4), the perturbation theory up to second order, and a numerical simulation of the exact timedependent BdG equations Eq. (S.9). We find that, for large modulation amplitudes, higher-order corrections become important because $A_{\mathbf{q}}$ [Eq. (A3)] is not a small parameter any more; more specifically, $\mathcal{J}_{2}(\alpha)$ is no longer small compared to the effective Bogoliubov dispersion $E_{\text {eff }}^{B}(\mathbf{q})$. We believe that this explains why the measured $q_{\text {mum }}^{x}$ curve in Fig. 4 deviates more from the zero-order BdG theory for large modulations amplitudes, i.e., $\alpha=1.78$, as compared to the rather good agreement observed for $\alpha=1.44$. We stress that the BdG analysis we present here does not rely on the inverse-frequency expansion, which is routinely used to determine the effective Hamiltonian in the context of a time-periodic Schrödinger equation.

\section{APPENDIX B: HARMONIC CONFINEMENT ALONG THE LATTICE DIRECTION}

In this Appendix, we analyze modifications to the translationally invariant Bogoliubov-de Gennes (BdG) theory (Sec. S.3 A in the Supplemental Material [52]) due to the harmonic trap along the lattice axis. We constrain the discussion to 1D systems for simplicity.

\section{Unbounded energy spectrum}

In most optical lattice experiments there is a harmonic confinement along the lattice axis, and therefore the energy spectrum of the system is no longer bounded from above. Figure 6 (orange dots) shows the energy spectrum of the effective time-averaged noninteracting Hamiltonian in the presence of the harmonic trap, and the corresponding 


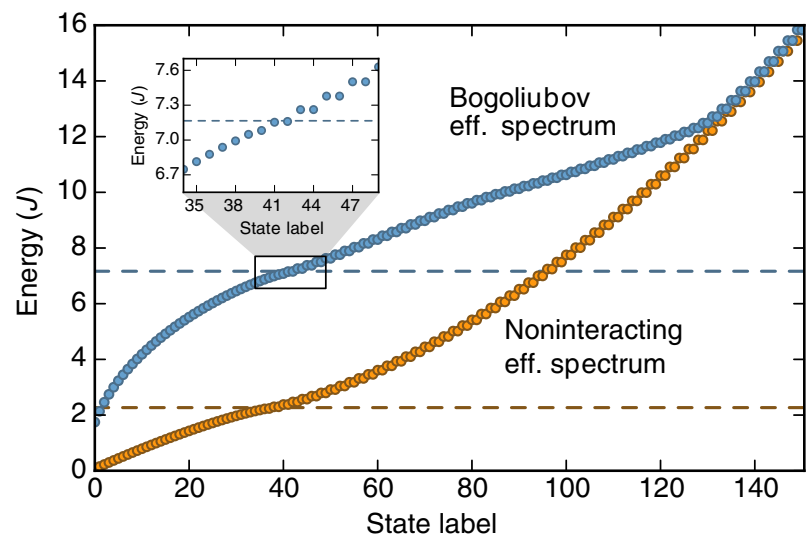

FIG. 6. Energy spectrum of the noninteracting time-averaged Hamiltonian (orange dots) and corresponding effective Bogoliubov dispersion (blue dots) for $\alpha=1.4$ and $g_{\max }=10.2 \mathrm{~J}$ in a harmonic trap with $\omega_{x}=0.26 J / \hbar$. The dashed lines indicate the respective bandwidths in the homogenous case, i.e., $\hbar \omega_{\text {sat }}=2.2 \mathrm{~J}$ (orange) and $\hbar \omega_{\text {sat }}=7.17 \mathrm{~J}$ (blue) according to Eq. (B1). Inset: As the energy reaches the homogenous bandwidth, the spectrum becomes quasidegenerate.

drive-renormalized Bogoliubov dispersion (blue dots). Details about the numerical simulations of the inhomogeneous system can be found in Sec. S4 B of the Supplemental Material [52]. Notice the occurrence of states above the Bogoliubov bandwidth (blue dashed line), which can be excited resonantly by the drive. In momentum space, these states occupy modes in the vicinity of $q^{x}=\pi / d$, and typically have weight over a finite range of momenta. Therefore, the presence of a weak harmonic confinement allows the system to absorb energy even for $\omega>\omega_{\text {sat }}$, where unconfined systems are shown to be stable [37]. As a result, we do not expect a truly stable parameter regime to exist in harmonically confined systems, even in the absence of transverse modes.

\section{Modified saturation frequency $\omega_{\text {sat }}$}

The expressions for the saturation frequency $\omega_{\text {sat }}$ and the effective bandwidth $W_{\text {eff }}$, derived using translationally invariant Bogoliubov theory, depend on the densityrenormalized interaction parameter $g$. To zeroth order (Appendix A), the effective bandwidth for the 1D lattice is obtained from Eq. (A2) by setting $\mathbf{q}^{\perp}=0$. In the presence of a harmonic confinement, the condensate profile obeys Thomas-Fermi theory and is no longer uniform, which induces a position dependence in $g \rightarrow g(x)$. Since the energy spectrum is unbounded, there is no natural energy bandwidth to use. Hence, we need to determine the appropriate value for $g$ used to compute the saturation frequency $\omega_{\text {sat }}$.

We observe a correlation between the energy at which the states in the Bogoliubov spectrum rapidly become quasidegenerate (cf. Fig. 6, inset) and the energy scale $\omega_{\text {sat }}\left(g_{\text {max }}\right)$ with $g_{\max }=\max _{x} g(x)$, where

$$
\hbar \omega_{\mathrm{sat}}(g, \alpha)=\sqrt{4 J_{\mathrm{eff}}(\alpha)\left[4 J_{\mathrm{eff}}(\alpha)+2 g\right]}
$$

The same scale also coincides with the energy, starting from which the Bogoliubov states attain a significant occupation of the $q^{x}=\pi / d$ mode. This suggests that, in the presence of a harmonic trap, the maximum value $g_{\max }$ can be used to estimate the saturation frequency $\omega_{\text {sat }}$.

To test this conjecture, we performed numerical BdG simulations of 1D lattices comparing the position of the most unstable mode with and without harmonic confinement. Indeed, we find a qualitative behavior similar to the homogeneous system, i.e., the momentum $q_{\text {mum }}^{x}(\omega)$ increases with $\omega$ until it saturates at $q_{\text {mum }}^{x} \approx \pi / d$. We can also quantitatively compare the $\alpha$ dependence of the saturation frequency $\omega_{\text {sat }}(\alpha)$ for the homogeneous and trapped systems as follows. (1) We fix a trap frequency and determine the value of $g_{\max }=U \max _{x} n_{\mathrm{TF}}(x)$ from the Thomas-Fermi profile of the condensate wave function $n_{\mathrm{TF}}(x)$. (2) We simulate the dynamics of a homogeneous system using the same value of $g$. If Eq. (B1) for $g=g_{\max }$ provides a correct description, this procedure will result in the same value of $\omega_{\text {sat }}$ for the trapped and homogeneous simulations by construction. The values of $\omega_{\text {sat }}(\alpha)$ are extracted numerically from the $q_{\text {mum }}^{x}$ versus $\omega$ curves for every fixed value of $\alpha$, as follows. (i) We compute numerically the momentum distribution profile of the Bogoliubov modes as a function of momentum $q^{x}$ and time $t$, evolved under the periodic drive. We do this for a grid of various $\alpha$ and $\omega$ points. (ii) We evolve the system for 20 driving cycles, which is enough to single out the most unstable mode that grows exponentially according to the $\mathrm{BdG}$ equations [52]. (iii) We extract the fastest growing mode $q_{\text {mum }}^{x}$ from the latest time slice for every point on the $(\alpha, \omega)$ grid. (iv) For every fixed $\alpha$, we determine the saturation frequency, by finding the frequency for which $q_{\text {mum }}^{x}$ reaches $\pi / d$ for the first time upon increasing $\omega$.

Figure 7 shows that the behavior of the saturation frequency $\omega_{\text {sat }}(\alpha)$ agrees with analytic predictions of Eq. (B1) for $g=g_{\max }$. We observe that the agreement gradually becomes limited in the regime of large $\alpha$ where the effective kinetic energy of the system is parametrically reduced for strong interactions $g$. This regime of large $\alpha$ and large $g / J$ is precisely where higher-order corrections to Eq. (A3) become pronounced (see the discussion in Appendix A). This explains the observed mismatch in the $g / J=10.2$ curves at large $\alpha$ in Fig. 7 .

\section{Modified BdG instability rates}

In Fig. 8 we show numerically evaluated instability rates of 1D lattice models using BdG simulations. We compare the drive frequency dependence for the inhomogeneous versus homogeneous lattices. Ideally, a homogeneous 1D system becomes stable for $\omega>\omega_{\text {sat }}$, since there are no states above the lattice bandwidth [75]. The simulation reveals that the presence of the harmonic trap does not lead 


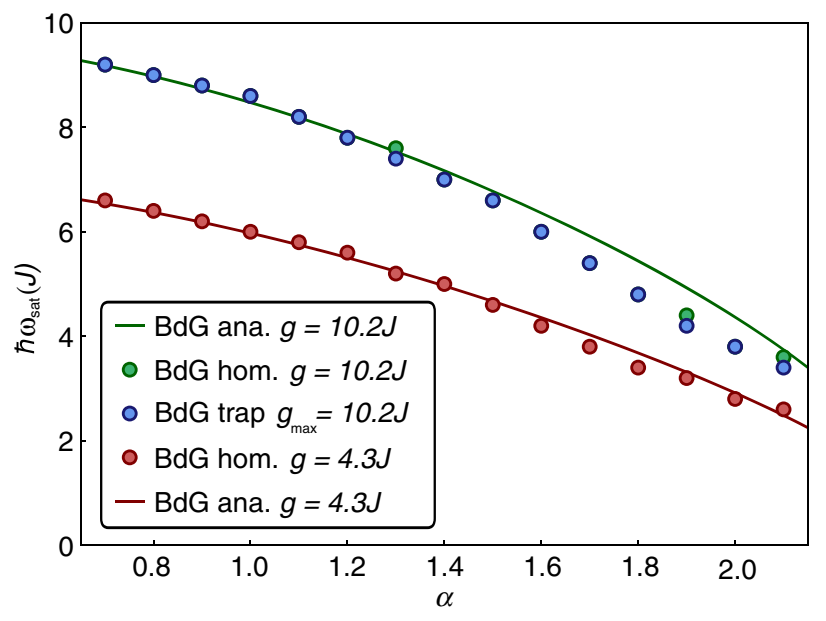

FIG. 7. Numerical simulation of the saturation frequency $\omega_{\text {sat }}$ versus $\alpha$ (dots) compared to the $\mathrm{BdG}$ prediction (solid line) from Eq. (B1). The presence of a harmonic trap does not affect the saturation frequency significantly. A slight deviation from the $\mathrm{BdG}$ prediction is observed compared to the numerical simulation for large values of $\alpha \sim 2$ and sufficiently strong effective interaction $g / J$. The trapping frequency is $\omega_{x}=0.26 J / \hbar$, the system size is $L_{x}=201 d$, and the atom number is $N_{0}=1000$.

to any further shift in the position of $\omega_{\text {sat }}$, provided one compares a homogeneous system of interaction strength $g$ to a harmonically confined system with $g_{\max }=g$. In the latter case, $\omega_{\text {sat }}$ is approximately given by the Bogoliubov bandwidth evaluated at $g_{\max }$ (Fig. 7). For larger modulation frequencies $\omega$, the trapped system displays a distinctly different behavior compared to the homogeneous system.

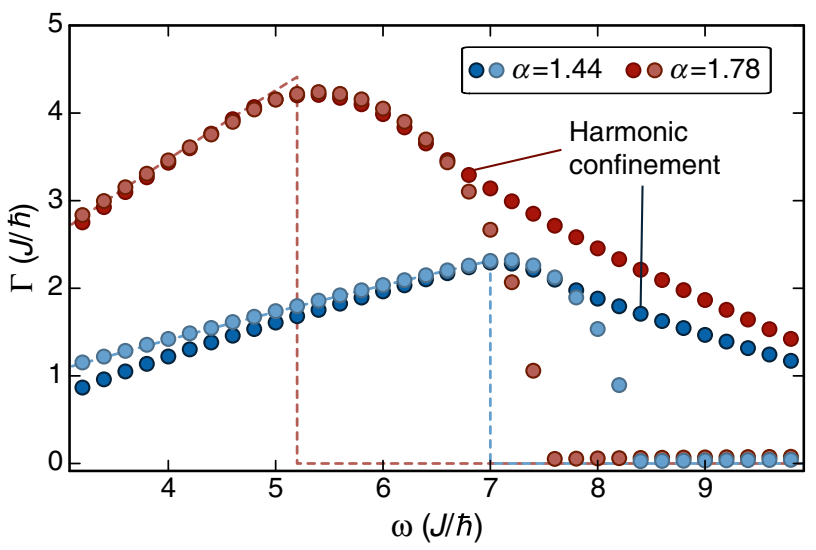

FIG. 8. Numerical simulations of the instability rate in homogeneous 1D lattices (light red and light blue, $g=10.2 J$ ) in comparison with the rates in $1 \mathrm{D}$ lattices with harmonic confinement (dark red and dark blue, $\omega_{x}=0.26 \mathrm{~J} / \hbar$ and $g_{\max }=10.2 \mathrm{~J}$ ) for two different driving parameters, $\alpha=1.4$ (blue) and $\alpha=1.8$ (red). The system parameters are the same as for the simulations in Fig. 7; i.e., $L_{x}=201 d$ and $N_{0}=1000$. The instability rate was extracted from an exponential fit to the numerical data from the last five out of 24 driving cycles of evolution, to make sure the maximally unstable mode dominates. The dashed vertical lines show the BdG predictions for $\omega_{\text {sat }}$ at $g=10.2 J$.
As already anticipated, due to the unbounded nature of the energy spectrum, there is no true stable (i.e., $\Gamma \equiv 0$ ) parameter regime and the confined system can always absorb energy via resonant processes. Nonetheless, we find a decrease of the instability rates with increasing drive frequency.

Because of the lack of translation invariance, it is not feasible to carry out an analytical calculation to determine the precise decay law $\Gamma(\omega)$ for $\omega>\omega_{\text {sat }}$ in the presence of the harmonic trap. However, assuming the local density approximation for a weak enough harmonic confinement and keeping in mind the unbounded structure of the spectrum, we can apply Eqs. (A5) and (A6) in all spatial regions of approximately constant density, according to which $\Gamma \propto \omega^{-1}$ for $\omega>\omega_{\text {sat }}$; while this does not predict the exact functional form of $\Gamma(\omega)$ observed in Fig. 8, it presents an approximate argument for the observed rate decay.

As a result, for trapped systems one can recover an approximately stable regime, where the instability rates are small compared to the duration of the experiment. An alternative way to mitigate the problem of nonvanishing rates at large drive frequencies in experiments could be provided by the use of uniform box traps $[65,66]$.

\section{APPENDIX C: INSTABILITY RATES}

Besides the position of the most unstable mode discussed in the main text, a characteristic property of parametric instabilities is the associated instability rates $\Gamma$, which after sufficiently long times are dominated by the growth of the occupation of the most unstable mode $n_{q_{\text {mum }}}$. Here, we present an experimental and numerical study of these rates.

\section{Influence of mode coupling}

In order to provide more insight on the time dependence of the instability rates found in the experiment [Fig. 3(a) and Fig. S2 in the Supplemental Material [52]], we performed numerical simulations (see Ref. [52] for details) on a homogeneous hybrid (translationally invariant) 2D system [76], composed of one lattice and one continuum direction, based on three different approximation methods [52]. (i) The linearized BdG equations, which capture the parametric instability at short times, i.e., before saturation effects, such as particle-number conservation, and nonlinear effects associated with the Gross-Pitaevskii equation, become significant. (ii) The weak-coupling conserving approximation (WCCA) [36], where particle-number conservation is restored, and which couples the condensate mode to the excitations to leading order in the interaction strength $U$. This method keeps track of the number of atoms scattered into finite-momentum modes, as well as the backaction of the Bogoliubov quasiparticles onto the condensate. The WCCA, however, does not capture collisions between quasiparticles. Hence, it does not offer any insight on the thermalization dynamics at longer times, 


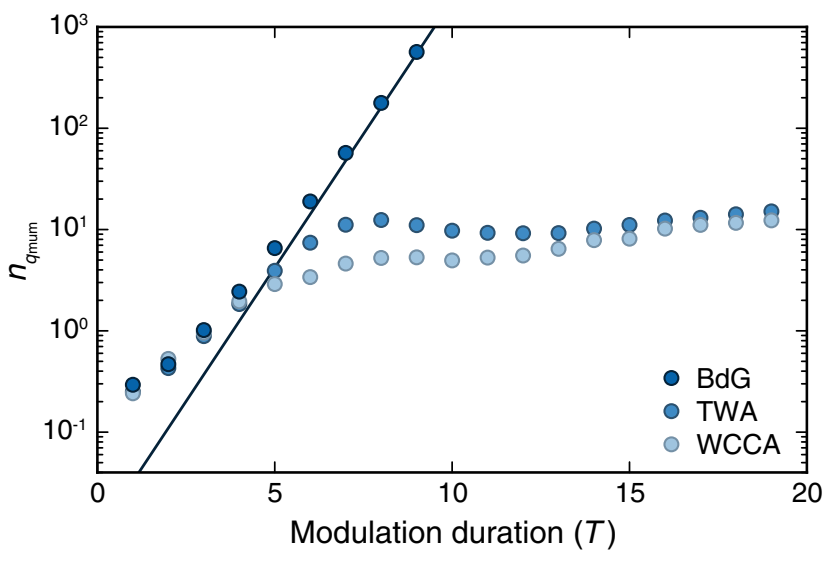

FIG. 9. Numerical simulations of the occupation of the most unstable mode $n_{q_{\text {mum }}}$ for a homogeneous 2D system (1D lattice and one continuous direction) for $g=9.52 \mathrm{~J}, \hbar \omega=9.25 \mathrm{~J}$, and $\alpha=1.44$. The solid line displays the rate obtained from the analytic formulas in Eqs. (A5) and (A6), which is in agreement with the BdG simulations (dark blue) for $t \gtrsim 5 T$. The TWA (blue) and WCCA (light blue) partially capture additional nonlinear effects, which result in a time-dependent instability rate.

during which the system heats up steadily to an infinitetemperature state. The BdG and WCCA approaches have already been compared in Ref. [37], and their validity at short times is further confirmed in this work by comparing their predictions to a third approach, namely (iii) the truncated Wigner approximation (TWA), which produces thermalizing dynamics. Even though quantum effects are only partially accounted for in the TWA semiclassical approximation, it has recently been demonstrated that classical Floquet systems thermalize in a very similar way to quantum models [55-57].

Figure 9 shows the time evolution of the occupation of the most unstable mode. In both the WCCA and TWA simulations, the obtained curves directly reflect the condensate depletion dynamics. At short times, we find an agreement between the three theoretical approaches, which further improves as one decreases the on-site interaction strength $U$, while keeping $g=n U$ fixed. At later times, the three approximations exhibit different behaviors: due to the lack of particle conservation, the BdG curves grow exponentially in an unphysical and indefinite manner. In contrast, the WCCA and TWA curves show clear manifestations of saturation effects, indicating that the instability rate is a truly time-dependent physical quantity. After an intermediate transient, all momentum modes are equally populated, which is expected for an infinite-temperature state that is reached at long times. These results offer a qualitative explanation for the saturation of the peak growth observed in the experiment, indicating the importance of saturation effects at intermediate modulation times, and further highlight the advantage of momentum-resolved measurements for revealing parametric instabilities (Fig. 4).

\section{Measured instability rates}

Motivated by the numerical analysis discussed above, we quantitatively study the exponential growth rate of the most unstable mode at short times, before saturation effects dominate the dynamics. To determine the saturation point of the amplitudes and the instability rates from the experimental data, we fit a piecewise function consisting of a linear and a constant part to the logarithm of the peak amplitude $A^{x}$ [52]. In BdG theory, the peak amplitudes along the $x$-lattice direction grow in time according to $A^{x} \propto e^{2 \Gamma t}$. Hence, from the fitted slopes $m$ (in the regime $t<t_{s}$ ), we extract the instability rates as $\Gamma=m / 2$ and average over the left and right peak. In Fig. 10, we show a comparison between the experimentally observed rates and the theoretical expectation based on the analytical BdG predictions [Eqs. (A5) and (A6)]. We further display the long-time heating rates, which have shown to be captured by Floquet Fermi's golden rule (FFGR) in Ref. [27]. For completeness, the relevant equations are summarized in the subsequent section.

We find that the experimental rates (dots in Fig. 10) are of similar magnitude as compared to the BdG predictions and about several orders of magnitude larger than the FFGR predictions [32]. The inset of Fig. 10 shows all rates on a linear scale, to illustrate the dependence on the modulation parameters. From BdG theory, it is expected that the rates have a maximum at the saturation frequency $\omega_{\text {sat }}$ (where $\hbar \omega$ equals the effective bandwidth). This maximum value is expected to increase with larger modulation amplitude.

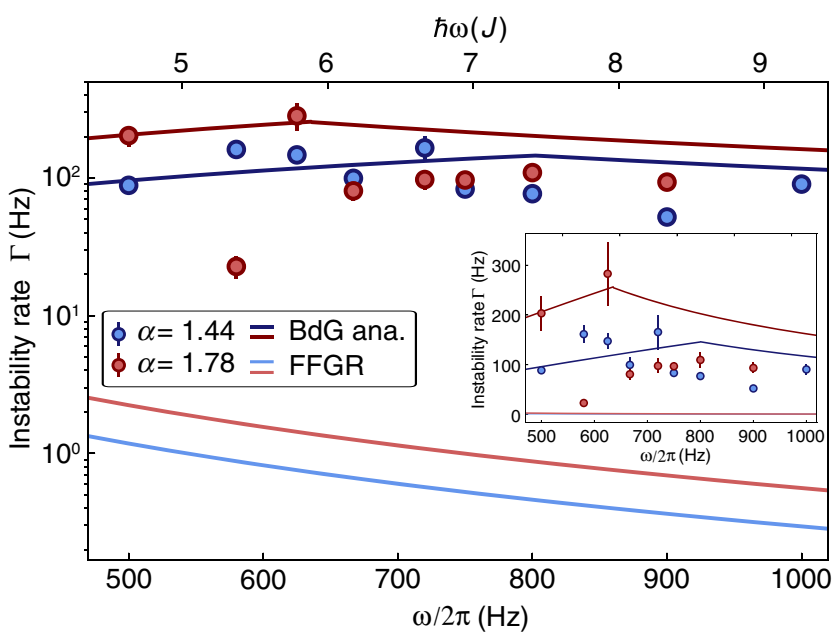

FIG. 10. Instability rates extracted from linear fits to the logarithmic peak amplitudes as a function of modulation time for $\alpha=1.44$ and $\alpha=1.78$. The dark solid lines are the theoretical rates calculated from Eqs. (A5) and (A6) for $g=11.5 \mathrm{~J}$, and the bright solid lines are a FFGR calculation for the same modulation parameters according to Eq. (C1). The inset shows the same data on a linear scale to reveal the frequency dependence of the measured rates. Error bars indicate the standard deviation. 
Although the measured rates lie in the same order of magnitude as the $\mathrm{BdG}$ rates, the $\mathrm{BdG}$-predicted parameter dependence is not directly confirmed by the measurements. Given the narrow window determined by $t_{s}$ during which the most unstable mode dominates, this is not surprising. More importantly, the discrepancy between the measured rates and the FFGR predictions shows that we are indeed probing the system on sufficiently short timescales, where instabilities are mostly driven by coherent processes.

\section{Floquet Fermi's golden rule}

Heating on long timescales dominated by incoherent processes is well described by Floquet Fermi's golden rule (FFGR) as studied in detail in Ref. [27]. The exponential decay of the BEC atoms caused by the modulated lattice can be described as

$$
\dot{N}(t)=-\Gamma_{\mathrm{FFGR}} N(t),
$$

where $\Gamma_{\mathrm{FFGR}}=\kappa N^{2 / 5}$. The loss rate $\kappa$ is independent of the atom number and given by

$\kappa(\alpha, \omega)=\frac{128}{105} \zeta^{-3 / 5}\left(\frac{15 \pi a_{s}}{8 d}\right)^{7 / 5}\left(\frac{\hbar \bar{\omega}}{E_{R}}\right)^{6 / 5} \frac{E_{R}}{\hbar} \sum_{l} \gamma_{l}$,

with

$$
\begin{aligned}
\zeta & =d \int\left|w_{0}(x)\right|^{4} d x, \\
\bar{\omega} & =\sqrt[3]{\omega_{r}^{2} \omega_{z}}, \\
\gamma_{l} & =6 u_{l} g\left(s_{l}\right)\left(\frac{\zeta J \mathcal{J}_{l}(\alpha)}{l \hbar \omega}\right)^{2}, \\
g\left(s_{l}\right) & =\left(\frac{1}{2}-\frac{1}{\pi} \arcsin \left(1-2 s_{l}\right)\right)-\frac{2 s_{l}+6}{3 \pi} \sqrt{s_{l}\left(1-s_{l}\right)}, \\
s_{l} & =\frac{l \hbar \omega}{8 J \mathcal{J}_{0}(\alpha)}, \\
u_{l} & =\left\{\begin{array}{ll}
0.75 & \text { for } l \text { even } \\
0.15 & \text { for } l \text { odd }
\end{array},\right.
\end{aligned}
$$

with $w_{0}(x)$ denoting the Wannier function of the lowest band and $\mathcal{J}_{\nu}$ being the $\nu$ th Bessel function of the first kind describing $\nu$-photon scattering processes. For the model parameters used in our study, the rates are dominated by processes up to second order, keeping contributions up to $l=2$. The FFGR rates are displayed in Fig. 10 (bright solid lines). It is evident that the FFGR rates are several orders of magnitude smaller than the BdG rates (dark solid lines). This failure of FFGR to capture the short-time heating dynamics can be traced back to the exponentially dominating short-time parametric instability effect which, in contrast to FFGR, is sustained by coherent dynamics. We note in passing that such parametric instabilities can only occur in bosonic systems (or, more precisely, in arbitrary systems with bosonic elementary excitations).

[1] N. Goldman and J. Dalibard, Periodically Driven Quantum Systems: Effective Hamiltonians and Engineered Gauge Fields, Phys. Rev. X 4, 031027 (2014).

[2] M. Bukov, L. D'Alessio, and A. Polkovnikov, Universal High-Frequency Behavior of Periodically Driven Systems: From Dynamical Stabilization to Floquet Engineering, Adv. Phys. 64, 139 (2015).

[3] A. Eckardt, Colloquium: Atomic Quantum Gases in Periodically Driven Optical Lattices, Rev. Mod. Phys. 89, 011004 (2017).

[4] D. Fausti, R. I. Tobey, N. Dean, S. Kaiser, A. Dienst, M. C. Hoffmann, S. Pyon, T. Takayama, H. Takagi, and A. Cavalleri, Light-Induced Superconductivity in a StripeOrdered Cuprate, Science 331, 189 (2011).

[5] Y. H. Wang, H. Steinberg, P. Jarillo-Herrero, and N. Gedik, Observation of Floquet-Bloch States on the Surface of a Topological Insulator, Science 342, 453 (2013).

[6] F. Mahmood, C.-K. Chan, Z. Alpichshev, D. Gardner, Y. Lee, P. A. Lee, and N. Gedik, Selective Scattering between Floquet-Bloch and Volkov States in a Topological Insulator, Nat. Phys. 12, 306 (2016).

[7] A. Cavalleri, Photo-Induced Superconductivity, Contemp. Phys. 59, 31 (2017).

[8] J. Zhang, P. W. Hess, A. Kyprianidis, P. Becker, A. Lee, J. Smith, G. Pagano, I. D. Potirniche, A. C. Potter, A. Vishwanath, N. Y. Yao, and C. Monroe, Observation of a Discrete Time Crystal, Nature (London) 543, 217 (2017).

[9] S. Choi, J. Choi, R. Landig, G. Kucsko, H. Zhou, J. Isoya, F. Jelezko, S. Onoda, H. Sumiya, V. Khemani, C. von Keyserlingk, N. Y. Yao, E. Demler, and M. D. Lukin, Observation of Discrete Time-Crystalline Order in a Disordered Dipolar Many-Body System, Nature (London) 543, 221 (2017).

[10] J. Struck, C. Ölschläger, R. Le Targat, P. Soltan-Panahi, A. Eckardt, M. Lewenstein, P. Windpassinger, and K. Sengstock, Quantum Simulation of Frustrated Classical Magnetism in Triangular Optical Lattices, Science 333, 996 (2011).

[11] M. Aidelsburger, M. Atala, S. Nascimbène, S. Trotzky, Y.-A. Chen, and I. Bloch, Experimental Realization of Strong Effective Magnetic Fields in an Optical Lattice, Phys. Rev. Lett. 107, 255301 (2011).

[12] H. Miyake, G. A. Siviloglou, C. J. Kennedy, W. C. Burton, and W. Ketterle, Realizing the Harper Hamiltonian with Laser-Assisted Tunneling in Optical Lattices, Phys. Rev. Lett. 111, 185302 (2013).

[13] G. Jotzu, M. Messer, R. Desbuquois, M. Lebrat, T. Uehlinger, D. Greif, and T. Esslinger, Experimental Realization of the Topological Haldane Model with Ultracold Fermions, Nature (London) 515, 237 (2014).

[14] M. Aidelsburger, M. Lohse, C. Schweizer, M. Atala, J. T. Barreiro, S. Nascimbène, N. R. Cooper, I. Bloch, and N. Goldman, Measuring the Chern Number of Hofstadter 
Bands with Ultracold Bosonic Atoms, Nat. Phys. 11, 162 (2015).

[15] N. R. Cooper, J. Dalibard, and I. B. Spielman, Topological Bands for Ultracold Atoms, Rev. Mod. Phys. 91, 015005 (2019).

[16] M. C. Rechtsman, J. M. Zeuner, Y. Plotnik, Y. Lumer, D. Podolsky, F. Dreisow, S. Nolte, M. Segev, and A. Szameit, Photonic Floquet Topological Insulators, Nature (London) 496, 196 (2013).

[17] M. Hafezi, S. Mittal, J. Fan, A. Migdall, and J. M. Taylor, Imaging Topological Edge States in Silicon Photonics, Nat. Photonics 7, 1001 (2013).

[18] T. Ozawa, H. M. Price, A. Amo, N. Goldman, M. Hafezi, L. Lu, M. C. Rechtsman, D. Schuster, J. Simon, O. Zilberberg, and I. Carusotto, Topological Photonics, Rev. Mod. Phys. 91, 015006 (2019).

[19] P. Roushan et al., Chiral Ground-State Currents of Interacting Photons in a Synthetic Magnetic Field, Nat. Phys. 13, 146 (2017).

[20] L. W. Clark, B. M. Anderson, L. Feng, A. Gaj, K. Levin, and C. Chin, Observation of Density-Dependent Gauge Fields in a Bose-Einstein Condensate Based on Micromotion Control in a Shaken Two-Dimensional Lattice, Phys. Rev. Lett. 121, 030402 (2018).

[21] F. Görg, K. Sandholzer, J. Minguzzi, R. Desbuquois, M. Messer, and T. Esslinger, Realization of Density-Dependent Peierls Phases to Engineer Quantized Gauge Fields Coupled to Ultracold Matter, Nat. Phys. 15, 1161 (2019).

[22] C. Schweizer, F. Grusdt, M. Berngruber, L. Barbiero, E. Demler, N. Goldman, I. Bloch, and M. Aidelsburger, Floquet Approach to $\mathbb{Z}_{2}$ Lattice Gauge Theories with Ultracold Atoms in Optical Lattices, Nat. Phys. 15, 1168 (2019).

[23] A. Lazarides, A. Das, and R. Moessner, Periodic Thermodynamics of Isolated Quantum Systems, Phys. Rev. Lett. 112, 150401 (2014).

[24] L. D'Alessio and M. Rigol, Long-Time Behavior of Isolated Periodically Driven Interacting Lattice Systems, Phys. Rev. X 4, 041048 (2014).

[25] G. Jotzu, M. Messer, F. Görg, D. Greif, R. Desbuquois, and T. Esslinger, Creating State-Dependent Lattices for Ultracold Fermions by Magnetic Gradient Modulation, Phys. Rev. Lett. 115, 073002 (2015).

[26] C. J. Kennedy, W. C. Burton, W. C. Chung, and W. Ketterle, Observation of Bose-Einstein Condensation in a Strong Synthetic Magnetic Field, Nat. Phys. 11, 859 (2015).

[27] M. Reitter, J. Näger, K. Wintersperger, C. Sträter, I. Bloch, A. Eckardt, and U. Schneider, Interaction Dependent Heating and Atom Loss in a Periodically Driven Optical Lattice, Phys. Rev. Lett. 119, 200402 (2017).

[28] C. Cabrera-Gutiérrez, E. Michon, M. Arnal, G. Chatelain, V. Brunaud, T. Kawalec, J. Billy, and D. Guéry-Odelin, Resonant Excitations of a Bose Einstein Condensate in an Optical Lattice, Eur. Phys. J. D 73, 170 (2019).

[29] M. Messer, K. Sandholzer, F. Görg, J. Minguzzi, R. Desbuquois, and T. Esslinger, Floquet Dynamics in Driven Fermi-Hubbard Systems, Phys. Rev. Lett. 121, 233603 (2018).

[30] T. Kitagawa, T. Oka, A. Brataas, L. Fu, and E. Demler, Transport Properties of Nonequilibrium Systems Under the
Application of Light: Photoinduced Quantum Hall Insulators without Landau Levels, Phys. Rev. B 84, 235108 (2011).

[31] S. Choudhury and E. J. Mueller, Transverse Collisional Instabilities of a Bose-Einstein Condensate in a Driven One-Dimensional Lattice, Phys. Rev. A 91, 023624 (2015).

[32] T. Bilitewski and N. R. Cooper, Scattering Theory for Floquet-Bloch States, Phys. Rev. A 91, 033601 (2015).

[33] T. Bilitewski and N. R. Cooper, Population Dynamics in a Floquet Realization of the Harper-Hofstadter Hamiltonian, Phys. Rev. A 91, 063611 (2015).

[34] M. Krämer, C. Tozzo, and F. Dalfovo, Parametric Excitation of a Bose-Einstein Condensate in a One-Dimensional Optical Lattice, Phys. Rev. A 71, 061602(R) (2005).

[35] C. E. Creffield, Instability and Control of a Periodically Driven Bose-Einstein Condensate, Phys. Rev. A 79, 063612 (2009).

[36] M. Bukov, S. Gopalakrishnan, M. Knap, and E. Demler, Prethermal Floquet Steady States and Instabilities in the Periodically Driven, Weakly Interacting Bose-Hubbard Model, Phys. Rev. Lett. 115, 205301 (2015).

[37] S. Lellouch, M. Bukov, E. Demler, and N. Goldman, Parametric Instability Rates in Periodically Driven Band Systems, Phys. Rev. X 7, 021015 (2017).

[38] T. Stöferle, H. Moritz, C. Schori, M. Köhl, and T. Esslinger, Transition from a Strongly Interacting $1 D$ Superfluid to a Mott Insulator, Phys. Rev. Lett. 92, 130403 (2004).

[39] H. Lignier, C. Sias, D. Ciampini, Y. Singh, A. Zenesini, O. Morsch, and E. Arimondo, Dynamical Control of MatterWave Tunneling in Periodic Potentials, Phys. Rev. Lett. 99, 220403 (2007).

[40] B. Wu and Q. Niu, Landau and Dynamical Instabilities of the Superflow of Bose-Einstein Condensates in Optical Lattices, Phys. Rev. A 64, 061603(R) (2001).

[41] M. Modugno, C. Tozzo, and F. Dalfovo, Role of Transverse Excitations in the Instability of Bose-Einstein Condensates Moving in Optical Lattices, Phys. Rev. A 70 , 043625 (2004).

[42] L. Fallani, L. De Sarlo, J. E. Lye, M. Modugno, R. Saers, C. Fort, and M. Inguscio, Observation of Dynamical Instability for a Bose-Einstein Condensate in a Moving $1 D$ Optical Lattice, Phys. Rev. Lett. 93, 140406 (2004).

[43] L. De Sarlo, L. Fallani, J. E. Lye, M. Modugno, R. Saers, C. Fort, and M. Inguscio, Unstable Regimes for a BoseEinstein Condensate in an Optical Lattice, Phys. Rev. A 72, 013603 (2005).

[44] N. Gemelke, E. Sarajlic, Y. Bidel, S. Hong, and S. Chu, Parametric Amplification of Matter Waves in Periodically Translated Optical Lattices, Phys. Rev. Lett. 95, 170404 (2005).

[45] G. K. Campbell, J. Mun, M. Boyd, E. W. Streed, W. Ketterle, and D. E. Pritchard, Parametric Amplification of Scattered Atom Pairs, Phys. Rev. Lett. 96, 020406 (2006).

[46] E. Michon, C. Cabrera-Gutiérrez, A. Fortun, M. Berger, M. Arnal, V. Brunaud, J. Billy, C. Petitjean, P. Schlagheck, and D. Guéry-Odelin, Phase Transition Kinetics for a Bose Einstein Condensate in a Periodically Driven Band System, New J. Phys. 20, 053035 (2018). 
[47] L. W. Clark, A. Gaj, L. Feng, and C. Chin, Collective Emission of Matter-Wave Jets from Driven Bose-Einstein Condensates, Nature (London) 551, 356 (2017).

[48] K. Staliunas, S. Longhi, and G. J. de Valcárcel, Faraday Patterns in Low-Dimensional Bose-Einstein Condensates, Phys. Rev. A 70, 011601(R) (2004).

[49] P. Engels, C. Atherton, and M. A. Hoefer, Observation of Faraday Waves in a Bose-Einstein Condensate, Phys. Rev. Lett. 98, 095301 (2007).

[50] H. Fu, L. Feng, B. M. Anderson, L. W. Clark, J. Hu, J. W. Andrade, C. Chin, and K. Levin, Density Waves and Jet Emission Asymmetry in Bose Fireworks, Phys. Rev. Lett. 121, 243001 (2018).

[51] Z. Zhang, K.-X. Yao, L. Feng, J. Hu, and C. Chin, Pattern Formation in a Driven Bose-Einstein Condensate, arXiv: 1909.05536.

[52] See Supplemental Material at http://link.aps.org/ supplemental/10.1103/PhysRevX.10.011030, which includes Refs. [53-60], for details on the data analysis, the full dataset (Figs. S2-S4), short descriptions of the different theoretical methods (BdG, WCCA, TWA), details on the numerical simulations, and a list of constants and model parameters.

[53] A. Chandran and S. L. Sondhi, Interaction-Stabilized Steady States in the Driven $O(N)$ Model, Phys. Rev. B 93, 174305 (2016).

[54] S. A. Weidinger and M. Knap, Floquet Prethermalization and Regimes of Heating in a Periodically Driven, Interacting Quantum System, Sci. Rep. 7, 45382 (2017).

[55] O. Howell, P. Weinberg, D. Sels, A. Polkovnikov, and M. Bukov, Asymptotic Prethermalization in Periodically Driven Classical Spin Chains, Phys. Rev. Lett. 122, 010602 (2019).

[56] S. Notarnicola, F. Iemini, D. Rossini, R. Fazio, A. Silva, and A. Russomanno, From Localization to Anomalous Diffusion in the Dynamics of Coupled Kicked Rotors, Phys. Rev. E 97, 022202 (2018).

[57] A. Rajak, R. Citro, and E. G. Dalla Torre, Stability and Pre-Thermalization in Chains of Classical Kicked Totors, J. Phys. A 51, 465001 (2018).

[58] P. B. Blakie, A. S. Bradley, M. J. Davis, R. J. Ballagh, and C. W. Gardiner, Dynamics and Statistical Mechanics of Ultra-Cold Bose Gases Using c-Field Techniques, Adv. Phys. 57, 363 (2008).

[59] A. Polkovnikov, Phase Space Representation of Quantum Dynamics, Ann. Phys. (Amsterdam) 325, 1790 (2010).

[60] D. A. W. Hutchinson, E. Zaremba, and A. Griffin, Finite Temperature Excitations of a Trapped Bose Gas, Phys. Rev. Lett. 78, 1842 (1997).

[61] M. Weinberg, C. Ölschläger, C. Sträter, S. Prelle, A. Eckardt, K. Sengstock, and J. Simonet, Multiphoton Interband Excitations of Quantum Gases in Driven Optical Lattices, Phys. Rev. A 92, 043621 (2015).

[62] M. Atala, M. Aidelsburger, M. Lohse, J. T. Barreiro, B. Paredes, and I. Bloch, Observation of Chiral Currents with Ultracold Atoms in Bosonic Ladders, Nat. Phys. 10, 588 (2014).
[63] H. Li-Chung, L. W. Clark, C. V. Parker, B. M. Anderson, and C. Chin, Roton-Maxon Excitation Spectrum of Bose Condensates in a Shaken Optical Lattice, Phys. Rev. Lett. 114, 055301 (2015).

[64] J.-R. Li, J. Lee, W. Huang, S. Burchesky, B. Shteynas, F. Ç. Top, A. O. Jamison, and W. Ketterle, A Stripe Phase with Supersolid Properties in Spin-Orbit-Coupled BoseEinstein Condensates, Nature (London) 543, 91 (2017).

[65] L. Corman, L. Chomaz, T. Bienaimé, R. Desbuquois, C. Weitenberg, S. Nascimbène, J. Dalibard, and J. Beugnon, Quench-Induced Supercurrents in an Annular Bose Gas, Phys. Rev. Lett. 113, 135302 (2014).

[66] N. Navon, A. L. Gaunt, R. P. Smith, and Z. Hadzibabic, Critical Dynamics of Spontaneous Symmetry Breaking in a Homogeneous Bose Gas, Science 347, 167 (2015).

[67] M. Babadi, M. Knap, I. Martin, G. Refael, and E. Demler, Theory of Parametrically Amplified Electron-Phonon Superconductivity, Phys. Rev. B 96, 014512 (2017).

[68] V. Peano, M. Houde, F. Marquardt, and A. A. Clerk, Topological Quantum Fluctuations and Traveling Wave Amplifiers, Phys. Rev. X 6, 041026 (2016).

[69] A. González-Tudela, C. L. Hung, D. E. Chang, J. I. Cirac, and H. J. Kimble, Subwavelength Vacuum Lattices and Atom-Atom Interactions in Two-Dimensional Photonic Crystals, Nat. Photonics 9, 320 (2015).

[70] J. Berges, K. Boguslavski, S. Schlichting, and R. Venugopalan, Basin of Attraction for Turbulent Thermalization and the Range of Validity of Classical-Statistical Simulations, J. High Energy Phys. 05 (2014) 054.

[71] P. Weinberg and M. Bukov, QuSpin: A Python Package for Dynamics and Exact Diagonalisation of Quantum Many Body Systems Part I: Spin Chains, SciPost Phys. 2, 003 (2017).

[72] P. Weinberg and M. Bukov, QuSpin: A Python Package for Dynamics and Exact Diagonalisation of Quantum Many Body Systems. Part II: Bosons, Fermions and Higher Spins, SciPost Phys. 7, 020 (2019).

[73] T. Boulier, J. Maslek, M. Bukov, C. Bracamontes, E. Magnan, S. Lellouch, E. Demler, N. Goldman, and J. V. Porto, Parametric Instabilities in a $2 \mathrm{D}$ Periodically-Driven Bosonic System: Beyond the Weakly-Interacting Regime, Phys. Rev. X 9, 011047 (2019).

[74] L. D. Landau and E. M. Lifshitz, Mechanics, Course of Theoretical Physics, Vol. 1 (Pergamon Press, Oxford, England, 1969).

[75] For homogeneous systems, it is known that the stability criterion can be shifted to slightly larger values due to the finite width of the parametric resonance [37], as can be seen in our simulations discussed in Appendix B (the dashed vertical lines in Fig. 8 show the position of the saturation frequency as predicted by $\mathrm{BdG}$ theory, which slightly precede the maximum of the numerical curves).

[76] We expect that the short-time dynamics should be reasonably well captured by a 2D model, as the confining potential is an order of magnitude stronger along the $z$ axis. 